\title{
Hints for the nematic pseudogap in the nearly optimally doped $\mathrm{La}_{2-x} \mathrm{Sr}_{x} \mathrm{CuO}_{4}$ superconductor
}

\author{
Marcin Matusiak $\odot,{ }^{1,2, *}$ Tadashi Adachi, ${ }^{3}$ Yoichi Tanabe $\odot,{ }^{4, \dagger}$ and Yoji Koike ${ }^{4}$ \\ ${ }^{1}$ Institute of Low Temperature and Structure Research, Polish Academy of Sciences, ulica Okolna 2, PL-50-422 Wroclaw, Poland \\ ${ }^{2}$ International Research Centre MagTop, Institute of Physics, Polish Academy of Sciences, Aleja Lotnikow 32/46, PL-02668 Warsaw, Poland \\ ${ }^{3}$ Department of Engineering and Applied Sciences, Sophia University, 7-1 Kioi-cho, Chiyoda-ku, Tokyo 102-8554, Japan \\ ${ }^{4}$ Department of Applied Physics, Tohoku University, 6-6-05 Aoba, Aramaki, Aoba-ku, Sendai 980-8579, Japan
}

(Received 8 April 2020; revised 10 July 2020; accepted 2 September 2020; published 17 September 2020)

\begin{abstract}
The in-plane anisotropy of the Seebeck $(S)$ and Nernst (v) coefficients of the nearly optimally doped cuprate superconductor $\mathrm{La}_{1.86} \mathrm{Sr}_{0.14} \mathrm{CuO}_{4}$ was measured under uniaxial pressure. Regardless of the qualitative differences between the $S(T)$ and $v(T)$ dependences, both the Seebeck and Nernst anisotropies evolve very similarly with temperature. Namely, they emerge slightly above the structural transition, then change their character, and eventually sign at the temperature close to the temperature of the pseudogap formation. We conclude that the pseudogap possibly breaks rotational symmetry and we indicate an important role played in this phase by small-angle inelastic scattering.
\end{abstract}

DOI: 10.1103/PhysRevResearch.2.032070

\section{INTRODUCTION}

Among the plethora of electronic orders emerging in the phase diagram of copper-based superconductors, the pseudogap is perhaps the most elusive one. Its presence was reported shortly after the discovery of high- $T_{c}$ superconductors $[1,2]$, and despite the enormous effort that was made to reveal the properties of the pseudogap, a physical origin of this phase is still being debated. The respective scenarios can be basically divided into two categories: these relating the pseudogap to the preformed, but not fully coherent, superconducting Cooper pairs [3-5] and those pointing at other types of electronic order [6-8] that are often claimed to compete with superconductivity [9-11]. Notably, different models for the formation of the pseudogap predict that the electron system in this state should break various symmetries [12-15]. The electronic nematicity, which appears to be common among various families of unconventional superconductors [15-21], breaks the rotational (but preserves translational) symmetry and has been recently suggested to be the true foundation of the pseudogap phase $[22,23]$.

Here, we provide experimental evidence that the pseudogap in a $\mathrm{La}_{2-x} \mathrm{Sr}_{x} \mathrm{CuO}_{4}$ high- $T_{\mathrm{c}}$ superconductor is in fact nematic. A single crystal of nearly optimally doped $\mathrm{La}_{2-x} \mathrm{Sr}_{x} \mathrm{CuO}_{4}$ with $x=0.141$ was subjected to uniaxial pressure applied within the $a b$ plane which allowed us to determine the in-plane anisotropy of the electrical resistivity as

\footnotetext{
*Corresponding author: matusiak@ magtop.ifpan.edu.pl

†Present address: Department of Applied Science, Okayama University of Science, 1-1 Ridai-cho, Kita-ku, Okayama 700-0005, Japan.

Published by the American Physical Society under the terms of the Creative Commons Attribution 4.0 International license. Further distribution of this work must maintain attribution to the author(s) and the published article's title, journal citation, and DOI.
}

well as the Seebeck and Nernst coefficients. The temperature dependences of the thermoelectrical phenomena indicate that the observed anisotropy is not directly related to the structural transition and a scenario explaining the formation of the pseudogap needs to include the presence of twofold in-plane anisotropy of the small-angle inelastic scattering.

\section{METHODS}

Single crystals of $\mathrm{La}_{2-x} \mathrm{Sr}_{x} \mathrm{CuO}_{4}$ (LSCO) were grown by the traveling-solvent floating-zone method under flowing $\mathrm{O}_{2}$ gas of 4 bars [24]. The as-grown single-crystalline rod was annealed in flowing $\mathrm{O}_{2}$ gas of 1 bar at $900{ }^{\circ} \mathrm{C}$ for $50 \mathrm{~h}$, cooled down to $500^{\circ} \mathrm{C}$ at a rate of $8{ }^{\circ} \mathrm{C} / \mathrm{h}$, kept at $500^{\circ} \mathrm{C}$ for $50 \mathrm{~h}$, and then cooled down to room temperature at a rate of $8^{\circ} \mathrm{C} / \mathrm{h}$. The $\mathrm{Sr}$ content of the sample was analyzed by inductively coupled-plasma optical-emission spectrometry (ICP-OES). The quality of the samples was checked by $\mathrm{x}$ ray back-Laue photography to be good. The samples were also checked by powder x-ray diffraction, in which no Bragg peaks due to impurities were observed. The full width at half maximum of the (006) rocking curve was $0.1^{\circ}-0.2^{\circ}$, which is comparable to that reported in the previous literature [24,25]. The distribution of the $\mathrm{Sr}$ content in a sample was also checked using an electron probe microanalyzer (EPMA) to be homogeneous within experimental accuracy.

For the transport measurements a squarelike plate with dimensions $2 \times 2 \times 0.1 \mathrm{~mm}^{3}$ was cut from the rod. The crystallographic $c$ axis was oriented along the shortest side of the sample, whereas the tetragonal $a$ axis was at $45^{\circ}$ with the sides of the face square. Such a sample was mounted between two clamps made of phosphor bronze and subjected to uniaxial pressure applied along its sides by a beryllium copper spring controlled with a stepper motor. For the resistivity measurements, the electrical contacts were placed at the corners of a sample and the orientations of the voltage and current leads were switched repetitively during the experiment. This 


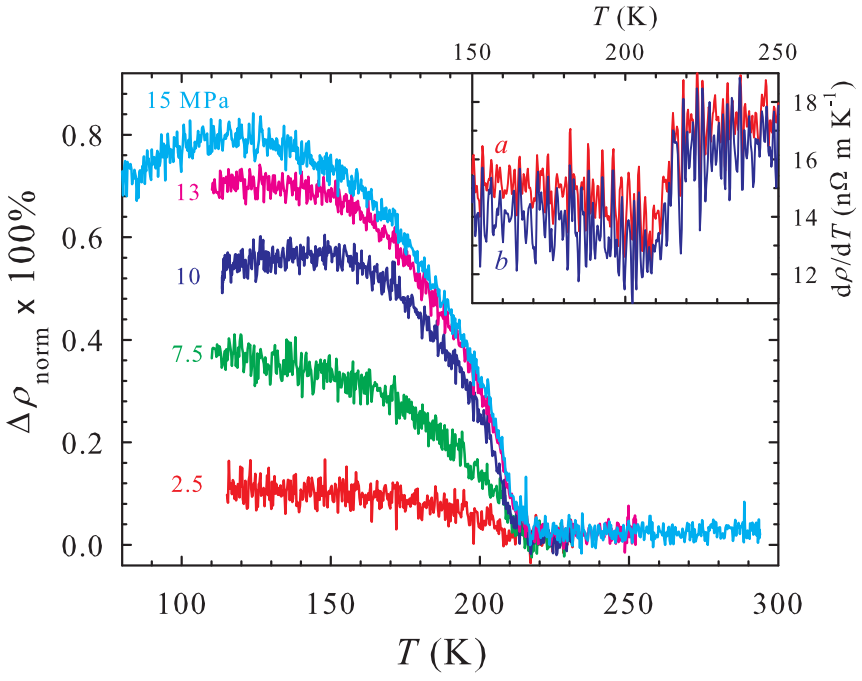

FIG. 1. Temperature dependences of the normalized in-plane resistivity anisotropy $\left(\Delta \rho_{\text {norm }}=\Delta \rho_{p}-\Delta \rho_{0}\right)$ for $\mathrm{La}_{1.86} \mathrm{Sr}_{0.14} \mathrm{CuO}_{4}$ under uniaxial pressures of approximately $2.5,7.5,10,13$, and $15 \mathrm{MPa}$. The inset presents the temperature derivative of the resistivity in the vicinity of the orthorhombic/tetragonal transition measured along the long $(a)$ and short $(b)$ crystallographic axes.

allowed the determination of the resistivity anisotropy using the Montgomery method [26].

The Seebeck and Nernst coefficients were measured along and across the strain direction in two separate runs where the magnetic field (parallel to the $c$ axis) was varied from -12.5 to $+12.5 \mathrm{~T}$. The temperature difference along a sample was determined using two Cernox thermometers as well as a calibrated in magnetic field constantan-chromel thermocouple attached to the sample through a few-millimeters-long and $100-\mu \mathrm{m}$-thick silver wires. Signal leads were made up of long pairs of $25-\mu \mathrm{m}$ phosphor bronze wires. The thermoelectrical coefficients measured in two experimental configurations differed within several percent in the high-temperature limit (due to slightly different geometrical factors), and were matched by applying a multiplicative correction factor close to 1 . More details about the experimental setup are given in the Supplemental Material [27].

\section{RESULTS}

In Fig. 1 the normalized in-plane anisotropy of the electrical resistivity $\left(\Delta \rho_{\text {norm }}\right)$ is plotted versus temperature for several values of the uniaxial pressure. Here, $\Delta \rho_{\text {norm }}$ is defined as the difference between the resistivity measured along the $a$ (long) and $b$ (short) orthorhombic crystallographic axes divided by the averaged value, $\Delta \rho=\left(\rho_{b}-\rho_{a}\right) /\left(\rho_{b}+\rho_{a}\right)$, with the offset from unstrained data. The resistivity anisotropy emerges at the transition to the orthorhombic state at about $T_{\text {str }} \approx 220 \mathrm{~K}$ that also manifests itself as a change of the $\rho_{a}(T)$ and $\rho_{b}(T)$ slopes visible as the steplike anomaly of $\partial \rho / \partial T$ presented in the inset in Fig. 1 . As expected, $\Delta \rho_{\text {norm }}$ in the orthorhombic phase rises with uniaxial pressure due to the increasing population of the domains oriented with the $b$ axis along the compressive strain. The resistivity anisotropy reaches a maximum of about $0.8 \%$ for $T \approx 120 \mathrm{~K}$ and then

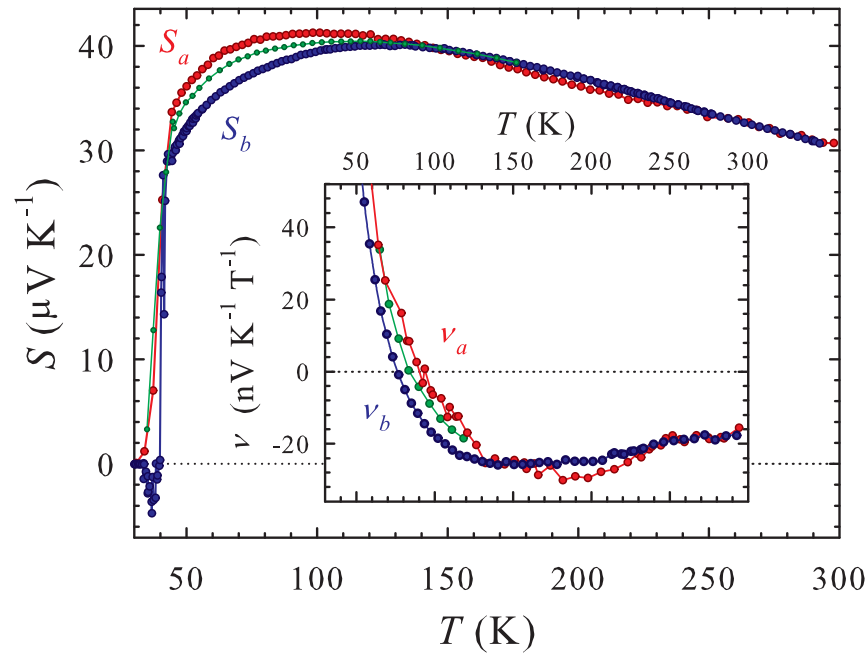

FIG. 2. Temperature dependences of the thermoelectric power for $\mathrm{La}_{1.86} \mathrm{Sr}_{0.14} \mathrm{CuO}_{4}$ measured along $a$ (red points) and $b$ (blue points) crystallographic axes under a uniaxial pressure of approximately $15 \mathrm{MPa}$. Green points that lie between $S_{a}$ and $S_{b}$ depict the Seebeck coefficient measured at ambient pressure. The inset presents the analogous temperature dependences of the Nernst coefficient.

drops with decreasing temperature. The latter effect possibly could be ascribed to the isotropic contribution to the electrical conductivity that results from Gaussian superconducting fluctuations occurring in LSCO in an extended range of temperatures [28]. An alternative explanation involves an increased relative contribution to the scattering of charge carriers from presumably isotropic collisions with defects, which, according to the Mathiessen rule, could also mask the anisotropic contribution. Noticeably, the relative difference between long and short crystallographic axes is still significantly smaller than $\Delta \rho_{\text {norm }}$, namely $(a-b) /(a+b) \times$ $100 \% \approx 0.16 \%$ at $100 \mathrm{~K}$ for $\mathrm{La}_{1.86} \mathrm{Sr}_{0.14} \mathrm{CuO}_{4}$, according to Ref. [29], $0.2 \%$ at $10 \mathrm{~K}$ [30], or even much less, i.e., about $0.01 \%$ at $100 \mathrm{~K}$ [31].

The temperature dependences of the Seebeck and Nernst coefficients ( $S$ and $v$, respectively) measured across (index $a$ ) and along (index $b$ ) the uniaxial pressure are presented in Fig. 2. The overall behaviors of $S(T)$ and $v(T)$ are analogous to those previously reported [32-34]. The clearly visible departure of the Nernst coefficient from its high-temperature linear temperature dependence, which occurs at $T \approx 190 \mathrm{~K}$ for $v_{a}$ and at $T \approx 170 \mathrm{~K}$ for $v_{b}$, was attributed to vortexlike excitations in the preformed phase [33] or Gaussian superconducting fluctuations [28] or the onset of a pseudogap [35]. To ensure that the detected anisotropies are not incidental, we also performed measurements for the unstrained sample, whose results are presented as green points in Fig. 2. Remarkably, both $S(T)$ and $v(T)$ dependences measured this way lie between the respective values obtained for the perpendicular and parallel uniaxial stress configurations.

Given the tiny difference in the size of the $a$ and $b$ lattice constants, it might be somewhat surprising to detect such a sizable difference between the $a$ - and $b$-indexed Seebeck and Nernst coefficients. While there is no obvious definition for a relative difference of the Nernst coefficient due to its 
sign change at $T \approx 80 \mathrm{~K}$, the relative Seebeck anisotropy $\left(S_{a}-S_{b}\right) /\left(S_{a}+S_{b}\right)$ at its maximum at $T \approx 50 \mathrm{~K}$ reaches $6 \%$, which is about two orders of magnitude higher than the relative crystallographic anisotropy. Therefore, the orthorhombicity cannot be the direct cause of the thermoelectric anisotropy but it rather serves as a weak external field that makes it possible to align the nematic domains. This in turn allows one to observe the macroscopic consequences of a tendency of the electronic system to self-organize in a way that breaks rotational symmetry.

In order to further investigate this phenomenon, the Seebeck and Nernst anisotropies $\left(\Delta S=S_{a}-S_{b}\right.$ and $\Delta v=v_{a}-$ $v_{b}$, respectively, divided by $T$ to account for temperature changes of entropy) are plotted along with the second temperature derivative of the electrical resistivity $\partial^{2} \rho / \partial T^{2}$ to highlight a correspondence between these three dependences. First, one can identify a maximum in $\partial^{2} \rho / \partial T^{2}$ at $T \approx 210 \mathrm{~K}$ which occurs at the tetragonal/orthorhombic transition [36] and slightly above the onset of this transition both Seebeck and Nernst coefficients deviate from zero. Subsequently, $\partial^{2} \rho / \partial T^{2}$ changes sign at $T^{*} \approx 170 \mathrm{~K}$, which marks the inflection point of $\rho(T)$. According to Ando et al. [36], this is related to the onset of the pseudogap and we use it to define $T^{*}$ which agrees well with the expected value for a given composition. Again, slightly above this temperature both $\Delta S(T) / T$ and $\Delta v(T) / T$ change their character, go through extrema (reaching $\Delta S_{\max }$ and $\Delta v_{\min }$, respectively), and consequently change sign at $T \approx 150 \mathrm{~K}$. This observation means that the thermoelectrical anisotropies caused by the structural transition and the emergence of the pseudogap have opposite signs. Interestingly, $\Delta \rho_{\text {norm }}$ does not exhibit any anomaly at $T^{*}$, while the evolution of $\Delta S(T) / T$ and $\Delta v(T) / T$ is very similar despite $S(T)$ and $v(T)$ being substantially different. This raises a question about a possible connection between the Nernst and Seebeck coefficients. Actually, these two are related, i.e., $v_{x}=-\frac{1}{B} \frac{\alpha_{y x}}{\sigma_{y y}}-S_{x} R_{\mathrm{H}} \sigma_{x x}$, where $\alpha_{y x}$ is the offdiagonal element of the Peltier tensor, $\sigma_{x x}$ and $\sigma_{y y}$ are the diagonal elements of the electrical conductivity tensor, and $R_{\mathrm{H}}$ is the Hall coefficient. However, the inset in Fig. 3 demonstrates that in the pseudogap phase the term containing $\Delta S$ is significantly smaller than $\Delta v$, hence the Nernst anisotropy cannot simply mirror the evolution of the Seebeck anisotropy.

Furthermore, despite the $S(T)$ and $v(T)$ dependences being different, the Seebeck and Nernst anisotropies plotted with the offsets $\Delta S_{\max }$ and $\Delta v_{\min }$, respectively, are practically identical in the entire temperature range. Figure 4 presents the $\left(\Delta S_{\max }-\Delta S\right) / T$ and $\left(\Delta v-\Delta v_{\min }\right) / T$ dependences, which keep rising with decreasing temperature, even when the resistivity anisotropy no longer increases below $T \approx 120 \mathrm{~K}$. Very similar behavior of $\Delta v / T(T)$ was observed in the iron-based superconductors $\mathrm{Ba}\left(\mathrm{Fe}_{1-x} \mathrm{Co}_{x}\right)_{2} \mathrm{As}_{2}$ [37], $\mathrm{Ca}\left(\mathrm{Fe}_{1-x} \mathrm{Co}_{x}\right)_{2} \mathrm{As}_{2}$ [20], FeTe [21], and also in a copper-based superconductor, $\mathrm{YBa}_{2} \mathrm{Cu}_{3} \mathrm{O}_{y}$ [38].

\section{DISCUSSION}

There are still controversies over the origin of the pseudogap, but also over basic properties of this enigmatic state. For instance, there is an ongoing debate over whether the pseudogap emerges in the phase transition [39,40], and if it is

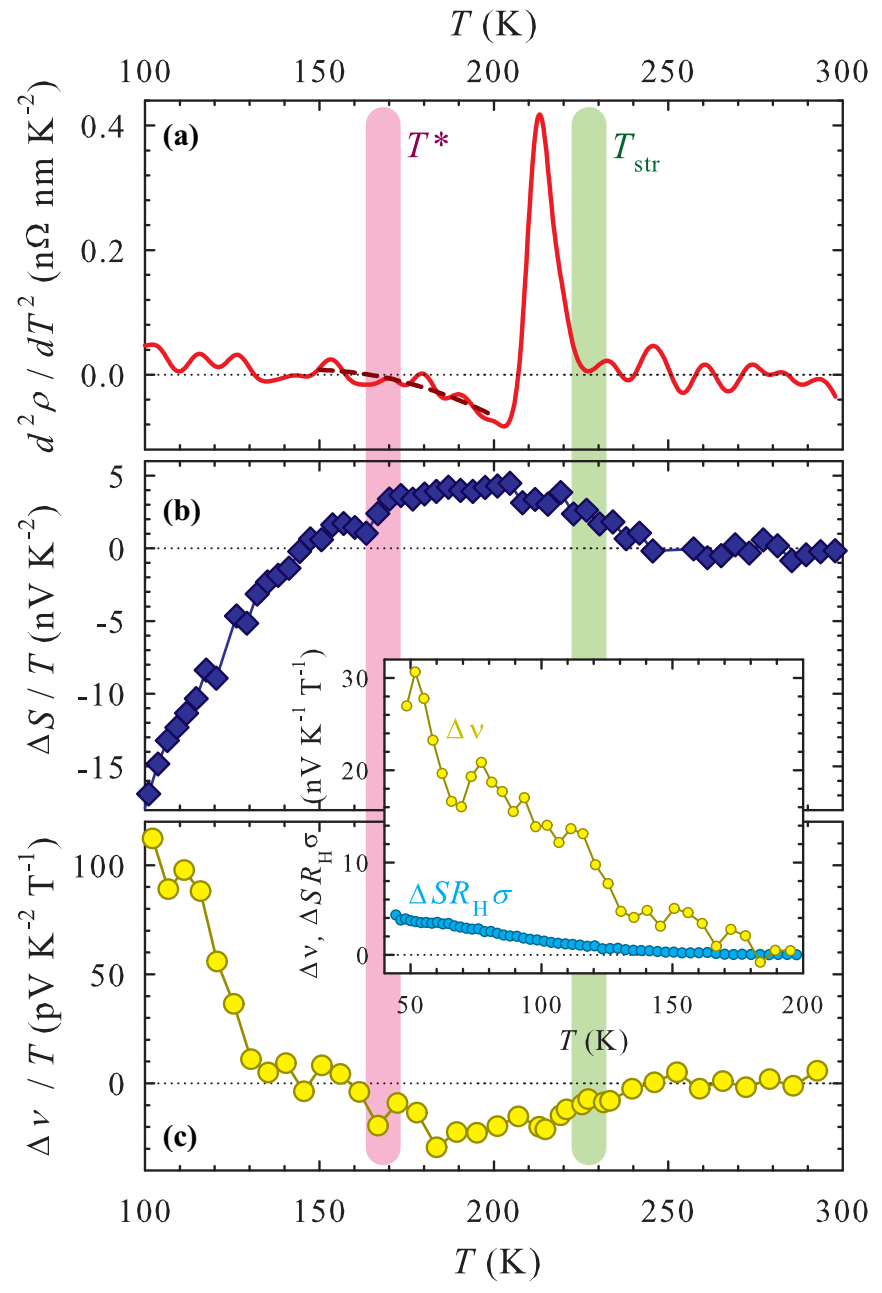

FIG. 3. Temperature dependences of (a) the second derivative of the resistivity, (b) the Seebeck anisotropy, and (c) the Nernst anisotropy for $\mathrm{La}_{1.86} \mathrm{Sr}_{0.14} \mathrm{CuO}_{4} . \partial^{2} \rho / \partial T^{2}$ is used to estimate temperatures of the structural transition at $T_{\text {str }} \approx 225 \mathrm{~K}$ marked with the vertical green shaded line on the right-hand side and the onset of the pseudogap at $T^{*} \approx 170 \mathrm{~K}$ marked with the vertical pink shaded line on the left-hand side. The inset presents a comparison of the $\Delta S R_{\mathrm{H}} \sigma$ term to $\Delta v\left(\Delta v \approx \frac{\Delta \alpha_{x y}}{B} \sigma-\Delta S R_{\mathrm{H}} \sigma\right)$, indicating that the former, as being significantly smaller, cannot account for the changes of the latter.

a "crossover" phenomenon [41] or rather a kind of fluctuating order [42]. It is also not clear which symmetries are broken below $T^{*}$, although some reports indicate that in this region the electron system breaks in-plane fourfold lattice symmetry $[15,23]$. On the other hand, studies of the transport properties in $\mathrm{YBa}_{2} \mathrm{Cu}_{3} \mathrm{O}_{y}$ point to a contribution from $\mathrm{Cu}-\mathrm{O}$ chains as a possible source of the anisotropy [38]. $\mathrm{La}_{2-x} \mathrm{Sr}_{x} \mathrm{CuO}_{4}$ differs from $\mathrm{YBa}_{2} \mathrm{Cu}_{3} \mathrm{O}_{y}$ in this respect, since its crystallographic structure lacks $\mathrm{Cu}-\mathrm{O}$ chains. The lattice obviously breaks fourfold symmetry in the low-temperature orthorhombic phase, but the crystallographic anisotropy is much smaller than the one observed in transport. Moreover, the electrical resistivity in thin films of $\mathrm{La}_{2-x} \mathrm{Sr}_{x} \mathrm{CuO}_{4}$, which were forced to retain tetragonal symmetry, still exhibited twofold symmetry 


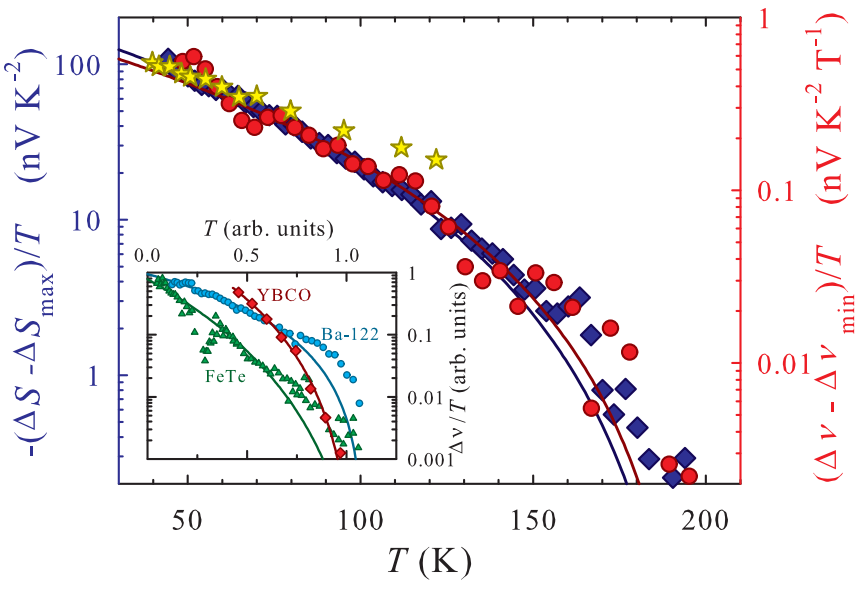

FIG. 4. Temperature dependences of the Seebeck (blue diamonds, left axis) and Nernst (red points, right axis) anisotropies shown in a semilogarithmic scale for $\mathrm{La}_{1.86} \mathrm{Sr}_{0.14} \mathrm{CuO}_{4}$. The plots include offsets $\left(\Delta S_{\max }\right.$ and $\Delta v_{\min }$, respectively) described in the text. Solid lines are fits to the Haller equation. The temperature dependence of the CDW peak height for $\mathrm{La}_{1.856} \mathrm{Sr}_{0.144} \mathrm{CuO}_{4}$ taken from Ref. [56] [normalized to the value of $-\left(\Delta S-\Delta S_{\max }\right) / T$ at $T=$ $50 \mathrm{~K}$ ] are superimposed on the plot as yellow stars. The inset presents exemplary temperature dependences of $\Delta v / T$ for $\mathrm{YBa}_{2} \mathrm{Cu}_{3} \mathrm{O}_{y}$ with a hole concentration $p=0.12$ (YBCO) [38], $\mathrm{Ba}\left(\mathrm{Fe}_{0.955} \mathrm{Co}_{0.045}\right)_{2} \mathrm{As}_{2}$ (Ba-122) [37], and FeTe [21], along with the respective Haller equation fits.

with the director loosely related to the orientation of the lattice [43].

Here, we show that while the onset of transport anisotropy correlates with the structural transition, $\Delta S(T) / T$ and $\Delta v(T) / T$ at lower temperatures change their character (and eventually sign), likely in response to the emergence of nematic order. Since the Seebeck and Nernst coefficients can be generally understood as a measure of the entropy flux [44], this indicates that the in-plane entropy transport becomes anisotropic (or, more specifically, suppressed along the uniaxial strain according to the signs of $\Delta S$ and $\Delta v$ ) below $T^{*}$. We would like to mention that we do not expect domain walls to play an important role in determining the in-plane anisotropy, since our sample at a uniaxial pressure of $P \approx 15 \mathrm{MPa}$ should be mostly detwinned. Early results of x-ray diffraction measurements on stressed $\mathrm{La}_{2-x} \mathrm{Sr}_{x} \mathrm{CuO}_{4}$ single crystals showed that for $P=1.5$ and $1.8 \mathrm{MPa}$ the detwinning ratio was about $90 \%$ [45] and 76\% [46], respectively.

There are several possible scenarios explaining anisotropic entropy flow in high- $T_{\mathrm{c}}$ superconductors. First, a contribution from vortexlike excitations [34] can be likely excluded, because this should mostly affect the transverse thermoelectric response. The superconducting fluctuations are also expected to influence the diagonal and off-diagonal elements of the Peltier tensor ( $\alpha_{x x}$ and $\alpha_{x y}$, respectively) differently [47]. We propose that the in-plane anisotropy can originate from an anisotropic scattering and since the resistivity and thermoelectrical anisotropies behave differently, we point at the small-angle inelastic scattering as a possible underlying mechanism. Such processes disturb mostly the entropy current, leaving the electrical transport almost unaffected [48,49]. Their abundance is characteristic for strongly correlated electron systems described, for example, as a hydrodynamic electron fluid [50,51], where the energy dissipation is dominated by small-angle electron-phonon scattering [52]. Alternatively, the electron-electron relaxation can be another source of momentum conserving collisions in the high- $T_{\mathrm{c}}$ superconductors [53], whereas in FeSe it can result from the interaction of charge carriers with anisotropic spin fluctuations [54]. Another possibility is the scattering of quasiparticles by charge density wave (CDW) fluctuations that get ordered in the weak strain field, and which were shown to be a source of small-angle inelastic scattering [55].

Noteworthy, CDW short-range order was recently detected in an extended range of temperatures for $\mathrm{La}_{2-x} \mathrm{Sr}_{x} \mathrm{CuO}_{4}$ [56] and electron nematicity may arise via a thermal or quantum phase transition from the proliferation of topological defects of the stripe (i.e., smectic) state [57]. A similar behavior was also observed in $\mathrm{NbSe}_{2}$, where short-range CDW survived beyond the phase coherence transition [58]. Using data from Ref. [56], we have superimposed the height of the CDW peak for similarly doped $\mathrm{La}_{2-x} \mathrm{Sr}_{x} \mathrm{CuO}_{4}(x=0.144)$ on the temperature dependences of the thermoelectrical anisotropies in Fig. 4. Evidently, all three quantities exhibit the same type of temperature dependence, and a similar, but not the same, slope.

The last subject we want to address is the temperature dependence of the Nernst coefficient that appears to be common for different unconventional superconductors, both in iron-based superconductors [20,21,37] and cuprates [38] (see the inset in Fig. 4). In Fig. 4 we fit the $\Delta S(T) / T$ and $\Delta v(T) / T$ data with the equation proposed to describe the nematic order parameter in liquid crystals [59], $\Delta v / T=$ $\alpha\left(1-\frac{T}{T_{H}}\right)^{\beta}$, where $\alpha$ is the proportional constant, $\beta$ is the pseudocritical exponent, and $T_{H}$ is a characteristic temperature somewhat lower than the actual temperature of the discontinuous nematic-isotropic transition. Interestingly, this fit seems to be universally applicable to the Nernst data from many different compounds, although the obtained values of $\beta$ are much different (e.g., $\beta \approx 2.6$ for $\mathrm{La}_{1.86} \mathrm{Sr}_{0.14} \mathrm{CuO}_{4}$ ) than values expected for liquid crystals, i.e., $\beta \approx 0.25$ [60].

\section{CONCLUSION}

The pseudogap is considered to be a key to understanding the mechanism leading to the formation of high- $T_{\mathrm{c}}$ superconductivity [61]. This belief has driven extensive studies aimed at the discovery of the true nature of the pseudogap, which led to the development of new experimental techniques, including measurements under uniaxial pressure. While there are still no definitive conclusions, there seems to be growing evidence that the pseudogap breaks rotational symmetry. One example is a very recent work by Ishida et al. [62] that reports nematic quantum criticality in lead-doped $\mathrm{Bi}_{2} \mathrm{Sr}_{2} \mathrm{CaCu}_{2} \mathrm{O}_{8+\delta}$. The presence of such a quantum critical point could be linked to superconductivity and so-called strange metallic behavior.

In general, the spontaneous tendency of an electron system to break rotational symmetry appears to be common in materials with strong electron correlations. In compounds with an orthorhombic crystalline structure, the observation of the macroscopic consequences of nematicity might be easier, 
because even a slight difference between the $a$ and $b$ lattice parameters provides a symmetry breaking field, which allows for the alignment of nematic domains [57]. However, this also can cause doubts about whether the detected anisotropy is not related to the orthorhombicity itself.

Our data show that the thermoelectric anisotropy in $\mathrm{La}_{1.86} \mathrm{Sr}_{0.14} \mathrm{CuO}_{4}$ indeed appears at the structural transition, but then it changes sign at $T^{*}$, which implies that the lowtemperature anisotropy is related to the pseudogap rather than a difference in the lattice parameters. Correspondingly, a recent study of $\mathrm{La}_{2-x} \mathrm{Sr}_{x} \mathrm{CuO}_{4}$ thin films under uniaxial strain shows that the nematicity is of an electronic origin, with very weak coupling to the crystal lattice [63]. Remarkably, while all the Seebeck, Nernst, and resistivity anisotropies emerge around the tetragonal/orthorhombic structural transition, only the thermoelectric ones seem to be sensitive to the onset of a pseudogap at $T^{*}$.
We conclude that the mechanism responsible for such a behavior is anisotropic small-angle scattering and we point at the short-range unidirectional charge density order as a possible root cause.

All of the relevant data that support the findings of this study are available from the corresponding author upon reasonable request.

\section{ACKNOWLEDGMENTS}

This work was supported by the Foundation for Polish Science through the IRA Programme cofinanced by EU within SG OP and by the National Science Centre (Poland) through Grant No. 2014/15/B/ST3/00357. T.A. was supported by the JSPS KAKENHI (No. JP19H01841 and No. JP20H05165). The authors declare no competing financial interests.
[1] J. G. Bednorz and K. A. Muller, Possible high $T_{\mathrm{c}}$ superconductivity in the Ba-La-Cu-O system, Z. Phys. B 64, 189 (1986).

[2] W. W. Warren Jr., R. E. Walstedt, G. F. Brennert, R. J. Cava, R. Tycko, R. F. Bell, and G. Dabbagh, Cu Spin Dynamics and Superconducting Precursor Effects in Planes Above $T_{\mathrm{c}}$ in $\mathrm{YBa}_{2} \mathrm{Cu}_{3} \mathrm{O}_{6.7}$, Phys. Rev. Lett. 62, 1193 (1989).

[3] V. Emery and S. Kivelson, Importance of phase fluctuations in superconductors with small superfluid density, Nature (London) 374, 434 (1995).

[4] M. Franz and A. J. Millis, Phase fluctuations and spectral properties of underdoped cuprates, Phys. Rev. B 58, 14572 (1998).

[5] P. Curty and H. Beck, Thermodynamics and Phase Diagram of High Temperature Superconductors, Phys. Rev. Lett. 91, 257002 (2003).

[6] C. M. Varma, Theory of the pseudogap state of the cuprates, Phys. Rev. B 73, 155113 (2006).

[7] Y. Li, V. Balédent, N. Barišić, Y. Cho, B. Fauqué, Y. Sidis, G. Yu, X. Zhao, P. Bourges, and M. Greven, Unusual magnetic order in the pseudogap region of the superconductor $\mathrm{HgBa}_{2} \mathrm{CuO}_{4+\delta}$, Nature (London) 455, 372 (2008).

[8] W. Tabis, Y. Li, M. Le Tacon, L. Braicovich, A. Kreyssig, M. Minola, G. Dellea, E. Weschke, M. J. Veit, M. Ramazanoglu, A. I. Goldman, T. Schmitt, G. Ghiringhelli, N. Barišić, M. K. Chan, C. J. Dorow, G. Yu, X. Zhao, B. Keimer, and M. Greven, Charge order and its connection with Fermi-liquid charge transport in a pristine high- $T_{\mathrm{c}}$ cuprate, Nat Commun. 5, 5875 (2014).

[9] R. Khasanov, T. Kondo, S. Strässle, D. O. G. Heron, A. Kaminski, H. Keller, S. L. Lee, and T. Takeuchi, Evidence for Competition between the Superconducting and the Pseudogap State in $(\mathrm{BiPb})_{2}(\mathrm{SrLa})_{2} \mathrm{CuO}_{6+\delta}$ from Muon-Spin Rotation Experiments, Phys. Rev. Lett. 101, 227002 (2008).

[10] T. Kondo, R. Khasanov, T. Takeuchi, J. Schmalian, and A. Kaminski, Competition between the pseudogap and superconductivity in the high- $T_{\mathrm{c}}$ copper oxides, Nature (London) $\mathbf{4 5 7}$, 296 (2009).

[11] T. Kondo, Y. Hamaya, D. A. Palczewski, T. Takeuchi, J. S. Wen, Z. J. Xu, G. Gu, J. Schmalian, and A. Kaminski, Disentangling Cooper-pair formation above the transition temperature from the pseudogap state in the cuprates, Nat. Phys. 7, 21 (2011).
[12] A. Kaminski, S. Rosenkranz, H. M. Fretwell, J. C. Campuzano, Z. Li, H. Raffy, W. G. Cullen, H. You, C. G. Olson, C. M. Varma, and H. Höchst, Spontaneous breaking of time reversal symmetry in the pseudogap state of a high- $T_{\mathrm{c}}$ superconductor, Nature (London) 416, 610 (2002).

[13] B. Fauqué, Y. Sidis, V. Hinkov, S. Pailhès, C. T. Lin, X. Chaud, and P. Bourges, Magnetic Order in the Pseudogap Phase of High- $T_{\mathrm{c}}$ Superconductors, Phys. Rev. Lett. 96, 197001 (2006).

[14] J. Xia, E. Schemm, G. Deutscher, S. A. Kivelson, D. A. Bonn, W. N. Hardy, R. Liang, W. Siemons, G. Koster, M. M. Fejer, and A. Kapitulnik, Polar Kerr-Effect Measurements of the High-Temperature $\mathrm{YBa}_{2} \mathrm{Cu}_{3} \mathrm{O}_{6+x}$ Superconductor: Evidence for Broken Symmetry near the Pseudogap Temperature, Phys. Rev. Lett. 100, 127002 (2008).

[15] R. Daou, J. Chang, D. LeBoeuf, O. Cyr-Choinière, F. Laliberté, N. Doiron-Leyraud, B. J. Ramshaw, R. Liang, D. A. Bonn, W. N. Hardy, and L. Taillefer, Broken rotational symmetry in the pseudogap phase of a high- $T_{\mathrm{c}}$ superconductor, Nature (London) 463, 519 (2010).

[16] M. J. Lawler, K. Fujita, J. Lee, A. R. Schmidt, Y. Kohsaka, C. K. Kim, H. Eisaki, S. Uchida, J. C. Davis, J. P. Sethna, and E.-A. Kim, Intra-unit-cell electronic nematicity of the high- $T_{\mathrm{c}}$ copperoxide pseudogap states, Nature (London) 466, 347 (2010).

[17] T.-M. Chuang, M. P. Allan, J. Lee, Y. Xie, N. Ni, S. L. Budko, G. S. Boebinger, P. C. Canfield, and J. C. Davis, Nematic electronic structure in the "parent" state of the iron-based superconductor $\mathrm{Ca}\left(\mathrm{Fe}_{1-x} \mathrm{Co}_{x}\right)_{2} \mathrm{As}_{2}$, Science 327, 181 (2010).

[18] J.-H. Chu, H.-H. Kuo, J. G. Analytis, and I. R. Fisher, Divergent nematic susceptibility in an iron arsenide superconductor, Science 337, 710 (2012).

[19] J. Wu, A. T. Bollinger, X. He, and I. Božović, Spontaneous breaking of rotational symmetry in copper oxide superconductors, Nature (London) 547, 431 (2017).

[20] M. Matusiak and M. Babij, Thermoelectric signature of the nematic phase in hole-doped iron-based superconductors, Phys. Rev. B 99, 174507 (2019).

[21] M. Matusiak and A. Krztoń-Maziopa, Nematicity in chalcogenide parent compound $\mathrm{Fe}_{1+y} \mathrm{Te}$ probed by thermoelectric measurements, J. Phys. Chem. Solids 139, 109311 (2020). 
[22] S. Mukhopadhyay, R. Sharma, C. K. Kim, S D. Edkins, M H. Hamidian, H. Eisaki, S.-.I. Uchida, E.-A. Kim, M. J. Lawler, A. P. Mackenzie, J. C. S. Davis, and K. Fujita, Evidence for a vestigial nematic state in the cuprate pseudogap phase, Proc. Natl. Acad. Sci. USA 116, 13249 (2019).

[23] H. Murayama, Y. Sato, R. Kurihara, S. Kasahara, Y. Mizukami, Y. Kasahara, H. Uchiyama, A. Yamamoto, E.-G. Moon, J. Cai, J. Freyermuth, M. Greven, T. Shibauchi, and Y. Matsuda, Diagonal nematicity in the pseudogap phase of $\mathrm{HgBa}_{2} \mathrm{CuO}_{4+\delta}$, Nat. Commun. 10, 3282 (2019).

[24] Y. Tanabe, T. Adachi, T. Noji, and Y. Koike, Superconducting volume fraction in overdoped regime of $\mathrm{La}_{2-x} \mathrm{Sr}_{x} \mathrm{CuO}_{4}$ : Implication for phase separation from magnetic-susceptibility measurement, J. Phys. Soc. Jpn. 74, 2893 (2005).

[25] S. Komiya, Y. Ando, X. F. Sun, and A. N. Lavrov, $c$-axis transport and resistivity anisotropy of lightly to moderately doped $\mathrm{La}_{2-x} \mathrm{Sr}_{x} \mathrm{CuO}_{4}$ single crystals: Implications on the charge transport mechanism, Phys. Rev. B 65, 214535 (2002).

[26] H. C. Montgomery, Method for measuring electrical resistivity of anisotropic materials, J. Appl. Phys. 42, 2971 (1971).

[27] See Supplemental Material at http://link.aps.org/supplemental/ 10.1103/PhysRevResearch.2.032070 for a description of the experimental techniques.

[28] I. Kokanović, J. R. Cooper, and M. Matusiak, Nernst Effect Measurements of Epitaxial $\mathrm{Y}_{0.95} \mathrm{Ca}_{0.05} \mathrm{Ba}_{2}\left(\mathrm{Cu}_{1-x} \mathrm{Zn}_{x}\right)_{3} \mathrm{O}_{y}$ and $\mathrm{Y}_{0.9} \mathrm{Ca}_{0.1} \mathrm{Ba}_{2} \mathrm{Cu}_{3} \mathrm{O}_{y}$ Superconducting Films, Phys. Rev. Lett. 102, 187002 (2009).

[29] Y. Watanabe, T. Hanaguri, T. Fukase, I. Tanaka, and H. Kojima, A low-temperature $\mathrm{x}$-ray diffraction study of structural phase transition in $\mathrm{La}_{1.86} \mathrm{Sr}_{0.14} \mathrm{CuO}_{4}$, Jpn. J. Appl. Phys. 29, 2763 (1990).

[30] B. Dabrowski, Z. Wang, K. Rogacki, J. D. Jorgensen, R. L. Hitterman, J. L. Wagner, B. A. Hunter, P. G. Radaelli, and D. G. Hinks, Dependence of Superconducting Transition Temperature on Doping and Structural Distortion of the $\mathrm{CuO}_{2}$ Planes in $\mathrm{La}_{2-x} \mathrm{M}_{x} \mathrm{CuO}_{4}(M=\mathrm{Nd}, \mathrm{Ca}, \mathrm{Sr})$, Phys. Rev. Lett. 76, 1348 (1996).

[31] N. Aomine, H. Yamada, Y. Soejima, A. Okazaki, I. Tanaka, and H. Kojima, Characterization of single crystals of high- $T_{\mathrm{c}}$ superconductor $\mathrm{La}_{2-x} \mathrm{Sr}_{x} \mathrm{CuO}_{4}$, Jpn. J. Appl. Phys. 32, 4959 (1993).

[32] J. S. Kim, B. H. Kim, D. C. Kim, and Y. W. Park, Thermoelectric power of $\mathrm{La}_{2-x} \mathrm{Sr}_{x} \mathrm{CuO}_{4}$ at high temperatures, Ann. Phys. (Leipzig) 13, 43 (2004).

[33] Z. A. Xu, N. P. Ong, Y. Wang, T. Kakeshita, and S. Uchida, Vortex-like excitations and the onset of superconducting phase fluctuation in underdoped $\mathrm{La}_{2-x} \mathrm{Sr}_{x} \mathrm{CuO}_{4}$, Nature (London) 406, 486 (2000).

[34] T. Fujii, T. Matsushima, T. Maruoka, and A. Asamitsu, Effect of stripe order strength for the Nernst effect in $\mathrm{La}_{2-x} \mathrm{Sr}_{x} \mathrm{CuO}_{4}$ single crystals, Physica C (Amsterdam) 470, S21 (2010).

[35] O. Cyr-Choinière, R. Daou, F. Laliberté, C. Collignon, S. Badoux, D. LeBoeuf, J. Chang, B. J. Ramshaw, D. A. Bonn, W. N. Hardy, R. Liang, J.-Q. Yan, J.-G. Cheng, J.-S. Zhou, J. B. Goodenough, S. Pyon, T. Takayama, H. Takagi, N. DoironLeyraud, and L. Taillefer, Pseudogap temperature $T^{*}$ of cuprate superconductors from the Nernst effect, Phys. Rev. B 97, 064502 (2018).

[36] Y. Ando, S. Komiya, K. Segawa, S. Ono, and Y. Kurita, Electronic Phase Diagram of High- $T_{\mathrm{c}}$ Cuprate Superconductors from a Mapping of the In-Plane Resistivity Curvature, Phys. Rev. Lett. 93, 267001 (2004).

[37] M. Matusiak, K. Rogacki, and T. Wolf, Thermoelectric anisotropy in the iron-based superconductor $\mathrm{Ba}\left(\mathrm{Fe}_{1-x} \mathrm{Co}_{x}\right)_{2} \mathrm{As}_{2}$, Phys. Rev. B 97, 220501(R) (2018).

[38] O. Cyr-Choinière, G. Grissonnanche, S. Badoux, J. Day, D. A. Bonn, W. N. Hardy, R. Liang, N. Doiron-Leyraud, and L. Taillefer, Two types of nematicity in the phase diagram of the cuprate superconductor $\mathrm{YBa}_{2} \mathrm{Cu}_{3} \mathrm{O}_{y}$, Phys. Rev. B 92, 224502 (2015).

[39] A. Shekhter, B. J. Ramshaw, R. Liang, W. N. Hardy, D. A. Bonn, F. F. Balakirev, R. D. McDonald, J. B. Betts, S. C. Riggs, and A. Migliori, Bounding the pseudogap with a line of phase transitions in $\mathrm{YBa}_{2} \mathrm{Cu}_{3} \mathrm{O}_{6+\delta}$, Nature (London) 498, 75 (2013).

[40] L. Zhao, C. A. Belvin, R. Liang, D. A. Bonn, W. N. Hardy, N. P. Armitage, and D. Hsieh, A global inversion-symmetry-broken phase inside the pseudogap region of $\mathrm{YBa}_{2} \mathrm{Cu}_{3} \mathrm{O}_{y}$, Nat. Phys. 13, 250 (2017).

[41] J. R. Cooper, J. W. Loram, I. Kokanović, J. G. Storey, and J. L. Tallon, Pseudogap in $\mathrm{YBa}_{2} \mathrm{Cu}_{3} \mathrm{O}_{6+\delta}$ is not bounded by a line of phase transitions: Thermodynamic evidence, Phys. Rev. B 89, 201104(R) (2014).

[42] C. V. Parker, P. Aynajian, E. H. da Silva Neto, A. Pushp, S. Ono, J. Wen, Z. Xu, G. Gu, and A. Yazdani, Fluctuating stripes at the onset of the pseudogap in the high- $T_{\mathrm{c}}$ superconductor $\mathrm{Bi}_{2} \mathrm{Sr}_{2} \mathrm{CaCu}_{2} \mathrm{O}_{8+x}$, Nature (London) 468, 677 (2010).

[43] J. Wu, A. T. Bollinger, X. He, and I. Božović, Spontaneous breaking of rotational symmetry in copper oxide superconductors, Nature (London) 547, 432 (2017).

[44] K. Behnia and H. Aubin, Nernst effect in metals and superconductors: A review of concepts and experiments, Rep. Prog. Phys. 79, 046502 (2016).

[45] S. Nakayama, Y. Maeno, M. Irie, M. Nohara, F. Nakamura, and T. Fujita, Anisotropy in thermal expansion of $\mathrm{La}_{2-x} \mathrm{Sr}_{x} \mathrm{CuO}_{4}$, Physica C 235-240, Part 2, 1283 (1994).

[46] F. Nakamura, M. Kodama, S. Nishizaki, Y. Tanaka, Y. Maeno, and T. Fujita, Structural and transport properties of detwinned crystals of $\mathrm{La}_{2-x} \mathrm{Sr}_{x} \mathrm{CuO}_{4}$, Physica C 235-240, Part 2, 1285 (1994).

[47] I. Ussishkin, S. L. Sondhi, and D. A. Huse, Gaussian Superconducting Fluctuations, Thermal Transport, and the Nernst Effect, Phys. Rev. Lett. 89, 287001 (2002).

[48] A. Jaoui, B. Fauqué, C. W. Rischau, A. Subedi, C. Fu, J. Gooth N. Kumar, V. Süß, D. L. Maslov, C. Felser, and K. Behnia, Departure from the Wiedemann-Franz law in $\mathrm{WP}_{2}$ driven by mismatch in $T$-square resistivity prefactors, npj Quantum Mater. 3, 64 (2018).

[49] N. W. Ashcroft and N. D. Mermin, Solid State Physics (Holt, Rinehart and Winston, New York, 1976).

[50] J. Gooth, F. Menges, N. Kumar, V. Süß, C. Shekhar, Y. Sun, U. Drechsler, R. Zierold, C. Felser, and B. Gotsmann, Thermal and electrical signatures of a hydrodynamic electron fluid in tungsten diphosphide, Nat. Commun. 9, 4093 (2018).

[51] A. Amoretti, M. Meinero, D. K. Brattan, F. Caglieris, E. Giannini, M. Affronte, C. Hess, B. Buechner, N. Magnoli, and M. Putti, Hydrodynamical description for magneto-transport in the strange metal phase of Bi-2200, Phys. Rev. Res. 2, 023387 (2020).

[52] R. N. Gurzhi, Hydrodynamic effects in solids at low temperature, Sov. Phys. Usp. 11, 255 (1968). 
[53] M. Reizer, Electron-electron relaxation in two-dimensional and layered superconductors, Phys. Rev. B 57, 1147 (1998).

[54] M. A. Tanatar, A. E. Böhmer, E. I. Timmons, M. Schütt, G. Drachuck, V. Taufour, K. Kothapalli, A. Kreyssig, S. L. Bud'ko, P. C. Canfield, R. M. Fernandes, and R. Prozorov, Origin of the Resistivity Anisotropy in the Nematic Phase of FeSe, Phys. Rev. Lett. 117, 127001 (2016).

[55] É. A. Pashitskil and V. I. Pentegov, Role of charge-density fluctuations and many-particle Coulomb correlations in the mechanism of high-temperature superconductivity of cuprate metal-oxide compounds, Low Temp. Phys. 27, 103 (2001).

[56] J.-J. Wen, H. Huang, S.-J. Lee, H. Jang, J. Knight, Y. S. Lee, M. Fujita, K. M. Suzuki, S. Asano, S. A. Kivelson, C.-C. Kao, and J.-S. Lee, Observation of two types of charge-density-wave orders in superconducting $\mathrm{La}_{2-x} \mathrm{Sr}_{x} \mathrm{CuO}_{4}$, Nat. Commun. 10, 3269 (2019).

[57] E. Fradkin, S. A. Kivelson, M. J. Lawler, J. P. Eisenstein, and A. P. Mackenzie, Nematic Fermi fluids in condensed matter physics, Annu. Rev. Condens. Matter Phys. 1, 153 (2010).
[58] K. Cho, M. Kończykowski, S. Teknowijoyo, M. A. Tanatar, J. Guss, P. B. Gartin, J. M. Wilde, A. Kreyssig, R. J. McQueeney, A. I. Goldman, V. Mishra, P. J. Hirschfeld, and R. Prozorov, Using controlled disorder to probe the interplay between charge order and superconductivity in $\mathrm{NbSe}_{2}$, Nat. Commun. 9, 2796 (2018).

[59] I. Haller, Thermodynamic and static properties of liquid crystals, Solid State Chem. 10, 103 (1975).

[60] A. Ranjkesh, M. Cvetko, J.-C. Choi, and H.-R. Kim, Phase and structural order in mixture of nematic liquid crystals and anisotropic nanoparticles, Phase Transitions 90, 423 (2017).

[61] S.-I. Uchida, Identifying the pseudogap in cuprates with a nematic phase, JPSJ News Comments 17, 07 (2020).

[62] K. Ishida, S. Hosoi, Y. Teramoto, T. Usui, Y. Mizukami, K. Itaka, Y. Matsuda, T. Watanabe, and T. Shibauchi, Divergent nematic susceptibility near the pseudogap critical point in a cuprate superconductor, J. Phys. Soc. Jpn. 89, 064707 (2020).

[63] A. T. Bollinger, Ze-B Wu, L. Wu, Xi He, I. Drozdov, J. Wu, I. Robinson, and I. Božović, Strain and electronic nematicity in $\mathrm{La}_{2-x} \mathrm{Sr}_{x} \mathrm{CuO}_{4}$, J. Supercond. Novel Magn. 33, 93 (2020). 